OPEN ACCESS

Citation: D. Benedetti (2020) L'uso dei media da parte dei musei nell'era della pandemia Covid-19: criticità e potenzialità. Media Education 11(2): 199-205. doi: 10.36253/me-9649

Received: August, 2020

Accepted: October, 2020

Published: December, 2020

Copyright: (c) 2020 D. Benedetti. This is an open access, peer-reviewed article published by Firenze University Press (http://www.fupress.com/me) and distributed under the terms of the Creative Commons Attribution License, which permits unrestricted use, distribution, and reproduction in any medium, provided the original author and source are credited.

Data Availability Statement: All relevant data are within the paper and its Supporting Information files.

Competing Interests: The Author(s) declare(s) no conflict of interest.
Best Practices

\section{L'uso dei media da parte dei musei nell'era della pandemia Covid-19: criticità e potenzialità}

\author{
Use of Media by Museums in the pandemic age of Covid-19: \\ problems and potentialities
}

\author{
Daniela Benedetti \\ Civico Museo Archeologico di Pegognaga \\ Museo Civico Antonio Parazzi di Viadana \\ E-mail: daniela.benedetti@tin.it
}

\begin{abstract}
The closing to the public due to the health emergency led museums to publish on the web a considerable amount of digital contents, in several cases self-produced. The modest diffusion in the Italian museums of a planned and conscious use of digital communication, resulting in part from the peculiarities of the national scene, revealed during the pandemic. It's appropriate move from the need to provide over the internet displays of resilience or contents alternatives to the possibility to go to the museum to a rethinking of the online offer, extremely challenging task for the several small and medium Italian museums, with few professional and economic resources.
\end{abstract}

Keywords: digital communication, museums, ICT, health emergency.

Riassunto. La chiusura al pubblico per l'emergenza sanitaria ha portato i musei a pubblicare una considerevole quantità di contenuti digitali sul web, in molti casi autoprodotti. La modesta diffusione dell'utilizzo programmato e consapevole della comunicazione digitale nei musei italiani, dovuta in parte alle peculiarità del panorama nazionale, è emersa durante la pandemia. Dalla necessità di offrire attraverso la rete manifestazioni di resilienza o contenuti alternativi alla possibilità di recarsi fisicamente in museo è opportuno passare per un ripensamento dell'offerta online, sfida particolarmente impegnativa per i numerosi piccoli e medi musei italiani, con poche risorse professionali ed economiche.

Parole chiave: comunicazione digitale, musei, ICT, emergenza sanitaria.

L’emergenza sanitaria causata dalla pandemia Covid-19 ha com'è noto costretto tutte le istituzioni culturali ad interrompere le attività con il pubblico, impedendo l'accesso fisico dei visitatori nei musei e bloccando tutte le attività finalizzate alla divulgazione e valorizzazione del patrimonio: percorsi didattico-laboratoriali con le scuole, eventi per famiglie, incontri, conferenze, rassegne. Tali iniziative rappresentano un'imprescindibile forma di comuni- 
cazione con il pubblico. Durante il lockdown i musei come tutti i luoghi di cultura - si sono attivati mettendo online una grande quantità di contenuti digitali. Nel disorientamento della fase più critica dell'emergenza sanitaria l'arte, la storia, la bellezza sono state sovente considerate, anche dai mezzi di comunicazione di massa, una sorta di antidoto allo smarrimento, e il patrimonio culturale è stato percepito talvolta come una rappresentazione simbolica dell'identità e della coesione di una comunità che si trovava a vivere questa drammatica esperienza collettiva. Qual è stata la risposta dei musei italiani e quali ricadute ha avuto?

\section{PREMESSA}

Le soluzioni utilizzate dalle istituzioni museali sono state eterogenee, rispecchiando la grande diversificazione del ricchissimo panorama italiano: nel censimento ISTAT per l'anno 2017 (2019a) dei 4.026 musei, gallerie o collezioni italiane, la prevalenza $(63,1 \%)$ risulta di proprietà pubblica e, fra questi, ben 2.067 appartengono ai Comuni. L'Italia ha dunque la peculiarità, rispetto ad altre nazioni, di avere un patrimonio disseminato sul territorio e costituito da strutture medio-piccole appartenenti in prevalenza ad enti locali. Nel 2017 un'esigua percentuale di musei e istituti statali appartenenti al Ministero competente, corrispondente al 9,8\% di musei, gallerie e collezioni, ha attratto il $44,3 \%$ dei visitatori; di contro quasi la metà delle organizzazioni museali censite, per la precisione il 41,3\%, ha registrato meno di 1000 visitatori annui. Le piccole dimensioni non possono ovviamente non avere ripercussioni sulle risorse professionali ed economiche e sulla capacità organizzativa delle istituzioni museali. A ciò si aggiunge un generalizzato ritardo nell'ambito della digitalizzazione e della comunicazione online. Dai rilevamenti del Report ISTAT per l'anno 2018 (2019b) risulta ad esempio che dei 4.908 musei, parchi archeologici, monumenti ed ecomusei censiti, solo il 10,4 $\%$ aveva una catalogazione scientifica digitale e solo il $9,9 \%$ offriva la possibilità di una visita virtuale. L'utilizzo delle tecnologie digitali e delle potenzialità offerte dal web in ambito italiano, sia pure in costante crescita, risulta ancora limitato per resistenza culturale, difficoltà ad adeguarsi ai ritmi rapidi e mutevoli delle ICT (Information and Communication Technologies) e per mancanza di competenze specifiche negli staff museali (Schnapp, 2012; Colombo, 2020).

Proprio su quest'ultimo aspetto il documento sulle professionalità del museo redatto da ICOM Italia nel 2017 esplicita l'importanza di figure professionali che si occupino del digitale dei musei con differenziate competenze e specifica che:

l'organizzazione della maggior parte delle strutture museali nazionali impone una minore frammentazione delle competenze e una sintesi della rosa dei professionisti digitali, mentre nelle grandi realtà culturali internazionali non solo queste figure sono presenti, ma sono parte dello staff di un intero dipartimento e, in alcuni casi, di centri di ricerca dedicati al digitale» (Jalla et al., 2017, p. 17) ${ }^{1}$.

\section{LA PRESENZA SUL WEB DEI MUSEI DURANTE L'EMERGENZA COVID}

Il ricorso al web durante l'emergenza sanitaria ha ovviamente risentito della limitata progettualità e consapevolezza dell'ambiente digitale di molte istituzioni museali. Musei, biblioteche, archivi, teatri hanno realizzato una grande quantità di prodotti digitali destinati alla pubblicazione e condivisione in rete. Il $90 \%$ circa dei musei che hanno risposto al questionario proposto da ICOM Italia ha creato contenuti ad hoc dopo la chiusura al pubblico ${ }^{2}$. I contributi sono stati innumerevoli, la loro analisi sistematica e puntuale, sia dal punto di vista qualitativo che quantitativo, richiederà tempo. In questa sede si propone una serie di esempi di istituzioni museali di differenti tipologie e dimensioni che, senza avere pretesa di esaustività, può offrire spunti di riflessione sulla presenza dei musei nel web.

La maggior parte dei contenuti digitali è costituita da approfondimenti su tematiche o elementi delle collezioni, spesso presentati in appuntamenti con cadenza regolare più o meno ravvicinata: le istituzioni museali hanno operato una selezione di manufatti, reperti e opere d'arte secondo i criteri di volta in volta ritenuti più opportuni - dai pezzi più noti o addirittura iconici dell'istituzione, a quelli meno conosciuti o curiosi - e li hanno resi disponibili sul web, soprattutto attraverso i canali social, in particolare Facebook e YouTube. A seconda del canale scelto e del target a cui si rivolgevano i musei hanno pubblicato post, fotografie, schede o, molto spesso, contributi video.

Le attività in ambito digitale durante la fase di pandemia hanno frequentemente posto l'accento sulla capacità narrativa dei musei, che si configurano sempre più non solo come spazio di conservazione ed esposizione, ma anche come luogo di racconto del passato, delle esperienze artistiche, delle discipline scientifiche ${ }^{3}$. Lo testimonia il ricorrere dei termini 'racconto' e 'storia' negli hashtag e nei titoli delle iniziative.

Sovente i contributi video hanno mostrato il direttore, membri dello staff o collaboratori del museo che 
illustravano una o più opere della collezione, a volte con un registro più divulgativo, come nelle "Pillole di archeologia" del Museo Archeologico e d'Arte della Marem$\mathrm{ma}^{4}$, in altri casi con modalità comunicative e linguaggio destinati ad un pubblico adulto, come nella campagna "\#UffiziDecameron"5 lanciata dagli Uffizi sui profili Instagram e Twitter per pubblicare foto, video e storie dedicate ai capolavori dell'istituzione o la serie "\#PoldiPezzolistories" del museo milanese ${ }^{6}$.

In alcuni casi le istituzioni hanno proposto sia video per bambini, dove prevaleva l'elemento ludico, sia interventi per adulti senza evidenziare i differenti destinatari dell'offerta, come "ogni cosa da noi è una storia...\#noidavoi" della Fondazione Ivan Bruschi ${ }^{7}$.

Il tema del racconto è, come abbiamo detto, ampiamente utilizzato dai musei, anche in casi in cui si mette in atto una spiegazione, più che una vera narrazione. Un esempio di storytelling, inteso come arte di raccontare in modo accattivante, utilizzando un linguaggio moderno e vicino ai destinatari della comunicazione, viene dal Museo delle Palafitte di Ledro, sede territoriale del Muse di Trento: i video autoprodotti dallo staff, intitolati "Quattro passi nella preistoria", dimostrano capacità di utilizzare inconsueti registri per condividere tematiche di preistoria ${ }^{8}$.

Alcuni importanti musei hanno scelto di illustrare proprio durante il periodo di forzata chiusura gli spazi e i materiali che normalmente non sono visibili al pubblico, come i depositi, e hanno fatto raccontare a curatori e tecnici aspetti inerenti la cura, la gestione del patrimonio o l'allestimento degli spazi museali: così hanno fatto i funzionari dei Musei Reali di Torino con l'iniziativa "I Musei Closed in. I Musei visti da dentro" e lo staff della Pinacoteca di Brera a Milano con "Appunti per una resistenza culturale"10.

Ricchissima ed estremamente diversificata l'offerta di alcune grandi istituzioni, spesso citate tra i musei italiani che da tempo curano e implementano la loro presenza sul web. Ne ricordiamo tre esempi che appartengono a tipologie diverse. Il MAXXI di Roma con "\#iorestoacasa" oltre a contributi sulle opere più significative della collezione (realizzate anche in LIS Lingua Italiana dei Segni

\footnotetext{
4 "Pillole di archeologia" è la definizione scelta per i contenuti pubblicati su Facebook e YouTube (\#bellodimaam, \#archeopilloledelMAAM).

${ }^{5} \mathrm{https} / /$ www.uffizi.it/magazine/uffizi-facebook-2020, 28/08/2020.

${ }^{6} \mathrm{https} / / /$ museopoldipezzoli.it/tutti-gli-eventi/poldipezzolistories/, 29/08/2020.

${ }^{7} \mathrm{http}: / / \mathrm{www}$. fondazioneivanbruschi.it/eventi/cosa-storia-noidavoi/, 29/08/2020.

${ }^{8} \mathrm{https} / / / \mathrm{www}$. youtube.com/watch? $\mathrm{v}=\mathrm{ICZu} 6 \mathrm{MFqIoA} \&$ list=PLS5GAt5gH hU_JXYcGMBQrFkADR3wPJKFp, 16/08/2020.

${ }^{9} \mathrm{https}: / /$ www.museireali-ereale.it/?cn-reloaded=1, 29/08/2020.

${ }^{10} \mathrm{https} / / /$ pinacotecabrera.org/brera-media/, 28/08/2020.
}

e con audio-descrizioni) ha proposto anche brevi video dedicati alle figure più significative dell'architettura e del design, proposte laboratoriali per bambini e famiglie, numerosi incontri con scrittori, registi, artisti, intellettuali su vari temi culturali e di attualità ${ }^{11}$. Ricco e diversificato per destinatari anche il panorama dei contributi del Museo Egizio di Torino ${ }^{12}$, dalle "Passeggiate del Direttore" alla serie "Istantanee dalla collezione", dal tour virtuale ai database sul patrimonio del Museo, dall'archivio delle conferenze trasmesse in streaming ai tutorial e racconti per bambini e famiglie. Molto nutrita anche l'offerta del Museo Nazionale della Scienza e della Tecnica di Milano con "Storie a porte chiuse": curatori, responsabili dei servizi educativi, giornalisti scientifici hanno offerto spunti di riflessione su argomenti scientifici, anche di stringente attualità come la pandemia, hanno raccontato personaggi o eventi della storia delle scoperte, hanno illustrato elementi delle collezioni ${ }^{13}$.

I musei hanno spesso dedicato ai bambini filmati, racconti, giochi e passatempo. Gli operatori didattici hanno proposto brevi tutorial con attività laboratoriali o di creatività, talvolta prendendo spunto da opere d'arte, libri o miti ${ }^{14}$. Oltre ai contenuti video possiamo citare giochi da fare online o da stampare ed utilizzare in formato cartaceo ${ }^{15}$.

L'interruzione delle attività con le scuole di ogni ordine e grado ha ovviamente rappresentato un elemento di forte criticità. Il pubblico scolastico rappresenta, soprattutto nei musei locali, la tipologia di visitatori più significativa. Quasi tutti i musei propongono una diversificata offerta didattica, che nella maggior parte dei casi prevede percorsi interattivi, visite animate, laboratori. Alcuni musei hanno preparato contenuti digitali per fornire uno strumento didattico in sostituzione dei servizi educativi di cui le scuole non potevano usufruire.

Tra questi la Fondazione Brescia Musei che, con l'iniziativa "Museo in classe. Visite virtuali e approfondimenti sulla storia e l'arte al servizio della scuola", ha proposto sei videolezioni di circa 30' dedicate ad altrettante parti della collezione: gli operatori didattici, ripresi

\footnotetext{
${ }^{11}$ https://www.maxxi.art/iorestoacasa/, 29/08/2020.

12 https://museoegizio.it/esplora/notizie/il-museo-egizio-a-casa-tua/, 28/08/2020

${ }^{13}$ https://www.museoscienza.org/it/storieaportechiuse, 29/08/2020.

14 A puro titolo di esempio tra i moltissimi "Gioca Museo da casa" (http://www.museicivicimonza.it/2020/04/27/iorestoacasagiocamuseoda-casa, 28/08/2020) e "Museo chiama casa - speciale bambini" proposto dall'Associazione Muse che opera nei musei di Firenze (http://musefirenze.it/museo-chiama-casa-speciale-bambini/, 28/08/2020).

15 L'iniziativa "Tempo di giochi" proposta dal Sistema Museale dell'Ateneo di Firenze, con contenuti differenziati per due fasce di età (https://www.sma.unifi.it/p609.html, 29/08/2020); semplici giochi da stampare disponibili in http://www.museopaleontologicomontevarchi. it/2020/03/19/visita-il-museo-da-casa/, 29/08/2020.
} 
nelle sale del museo, approfondiscono alcune tematiche storico-archeologiche e al termine suggeriscono delle semplici attività laboratoriali legate all'argomento trattato $^{16}$. La Fondazione del Museo Civico di Rovereto con "\#apertiperlascuola" ha realizzato 14 video sulla Preistoria e su diverse discipline scientifiche destinati alla scuola primaria ${ }^{17}$. Il MAP di Perfugas ha inserito sul sito ${ }^{18}$ una serie di video di approfondimento su tematiche legate alla preistoria destinati alle scuole, ma non solo, con il proposito di «accorciare le distanze tra museo e visitatore». L'offerta educativa del Museo Archeologico di Aquileia è articolata in una serie di video, della durata di alcuni minuti, dedicati ad un reperto o una tipologia di materiali; ad ogni contributo è associata una scheda stampabile, in cui si trovano i concetti fondamentali trattati nel video, il suggerimento di attività laboratoriali e l'immagine del reperto da incollare sulla linea del tempo, che può accogliere tutti i manufatti illustrati per ricostruire la storia del sito ${ }^{19}$.

Alcune istituzioni avevano già da tempo predisposto e messo online un'offerta di strumenti digitali per la didattica e la formazione. Si segnala ad esempio il Museo Galileo di Firenze che ha una diversificata e ricca serie di video didattici sulla storia della scienza, realizzati anche con importati collaborazioni, schede in pdf, attività da svolgere in classe ${ }^{20}$. A questo materiale già disponibile si è aggiunta, nell'ottica della didattica a distanza, la possibilità per le scuole di richiedere il supporto degli operatori con videolezioni svolte attraverso le piattaforme digitali dei singoli istituti. Anche "Senza titolo" con il Dipartimento educativo del Museo di Arte Moderna di Bologna ha proposto una serie di percorsi educativi a distanza per le scuole della città metropolitana di Bologna con quattro modalità di fruizione: incontri in videoconferenza, incontri in modalità Classroom, incontri in videoconferenza rivolti agli insegnanti, videolezioni inviate agli insegnanti da utilizzare come supporto alle lezioni a distanza ${ }^{21}$.

\section{CRITICITÀ E POTENZIALITÀ}

I contenuti proposti in rete dai musei durante il

\footnotetext{
${ }^{16} \mathrm{https}$ //didattica.bresciamusei.com/pag.asp? $\mathrm{n}=29 \& \mathrm{t}=$ Spazio+Scuola, 29/08/2020.

17 http://www.fondazionemcr.it/context.jsp? ID LINK=114890\&area=279, 29/08/2020.

${ }^{18} \mathrm{https} / / /$ www.sarundine.com/approfondimenti-video/, 29/08/2020.

19 https://museoarcheologicoaquileia.beniculturali.it/ ilmuseoacasa/\#page-content, 28/08/2020.

${ }^{20} \mathrm{https} / /$ www.museogalileo.it/it/museo/impara/online.html, 28/08/2020.

${ }^{21} \mathrm{https} / / / \mathrm{www}$. senzatitolo.net/in-corso/316/didattica_a_distanza_tra_ scuola_e_museo:_proposte_educative_in_digitale_per_larte_contemporanea_/, 28/08/2020.
}

lockdown, in particolare durante le fasi 1 e 2 , sono stati ovviamente realizzati in condizioni emergenziali, in molti casi autoprodotti con gli strumenti e le professionalità interne ai musei ${ }^{22}$. Gli esempi sopra menzionati, diversificati per esiti e intenti, rappresentano una piccolissima percentuale dell'enorme quantità di contributi pubblicati dai musei, grandi e piccoli, nel corso della pandemia; la chiusura forzata ha evidenziato il diffuso ritardo dei musei italiani in ambito digitale e le istituzioni che avevano investito e programmato la loro presenza e attività sul web in questo periodo hanno saputo dare una risposta puntuale e sistematica alle nuove esigenze. La comunicazione digitale richiede una programmazione su tempi lunghi, una strategia condivisa e coerente con la missione e la visione del museo, figure professionali competenti nelle ICT, nella progettazione e gestione dei contenuti, esperti in digital video. Ciò naturalmente rappresenta una sfida particolarmente difficile per i musei di piccole e medie dimensioni che, come detto, costituiscono una parte estremamente considerevole del panorama italiano; essi potrebbero trarre grandi vantaggi in termini di visibilità, valorizzazione, acquisizione di nuovo pubblico dal web, in particolare quelli che geograficamente sono esclusi dai flussi turistici di massa, ma sono penalizzati dai molti limiti nell'organizzazione e nelle risorse professionali ed economiche ${ }^{23}$.

Un ambito che richiede una particolare attenzione è rappresentato dal rapporto con le scuole; se da un lato i servizi educativi dei musei predispongono percorsi differenziati, che privilegiano un approccio pedagogicodidattico hands-on, attività multisensoriali, coinvolgenti e cooperative, dall'altro non si registra in Italia una pari diffusione in rete di strumenti digitali didattici efficaci ${ }^{24}$. Il web potrebbe essere la sede opportuna non solo per fornire materiali utili per la formazione pre-visita e postvisita (Dipace, 2012; Caprara, Colombi \& Scala, 2018, pp. 81-83), ma anche un'offerta didattica creata per una fruizione differente e non in competizione con quella che gli studenti possono vivere fisicamente visitando i musei.

È auspicabile, quando non essenziale, il coinvolgimento di differenti professionalità per realizzare percorsi efficaci nell'ottica dell'edutainment, neologismo nato

\footnotetext{
${ }^{22}$ Dai risultati del questionario proposto ai Musei da ICOM Italia sopra menzionato risulta che quasi l' $84 \%$ dei musei non ha allocato budget per la comunicazione digitale dopo la chiusura, utilizzando personale interno e/o di struttura.

${ }^{23}$ I musei medio-piccoli possono ricorrere, come spesso già avviene per altri servizi essenziali, alla condivisione di figure professionali. La mancanza di risorse interne naturalmente rende più difficoltoso mantenere l'aderenza della comunicazione online con le caratteristiche e l'identità dell'istituzione (sul tema Panzeri, 2010, p. 93).

${ }^{24}$ Spunti bibliografici sulla tecnologia nella didattica museale e nell'apprendimento a distanza in Bonacasa, 2011, pp. 146-151; Lin \& Gregor, 2006.
} 
dalla fusione dei termini inglesi education and entertainment con il quale si indicano le attività educative svolte coniugando insegnamento e svago. Da tempo il gioco e l'elemento emozionale sono entrati nella didattica museale, con maggiore lentezza l'edutainment viene introdotto nell'offerta web dei musei italiani (Valentino \& Delli Quadri, 2004).

L'emergenza sanitaria ha indirizzato i musei non solo a produrre materiali disponibili offline, come contributi video o schede, ma anche a proporre attività online con esperti, operatori didattici e mediatori scientifici; questa interazione "a distanza" può sicuramente essere sviluppata, ma per essere efficace necessita di una riformulazione della modalità di gestione del momento didattico ${ }^{25}$.

Le esperienze fatte nel periodo pandemico possono essere punto di partenza per sviluppare diverse attività che, favorendo la conoscenza del patrimonio culturale, trovano corrispondenza con le finalità del Protocollo d'intesa tra MIUR e MIBAC in materia di applicazione delle tecnologie per l'istruzione e la diffusione della cultura (Ciocca, 2006).

In particolare, possono avere ricadute positive:

- la digitalizzazione del patrimonio museale, amplificando il materiale informativo a disposizione di studenti e docenti;

- la valorizzazione di reperti, opere d'arte, manufatti e strumenti scientifici che di solito non sono inseriti nei percorsi educativi del museo, ma che possono rappresentare interessanti risorse didattiche;

- proporre percorsi digitali che, svincolati dai limiti fisici, possono mettere in relazione beni del museo fisicamente, culturalmente o cronologicamente non vicini, o creare collegamenti con il territorio, favorendo la coscienza del patrimonio culturale diffuso;

- suggerire attività di tipo collaborativo che, analogamente con quanto spesso si propone in museo, consenta agli studenti di svolgere giochi di gruppo o problem solving ${ }^{26}$;

- implementare la preparazione di contenuti digitali (podcast, filmati, videoclip) dedicati al racconto del patrimonio; la narrazione infatti può generare interesse e conoscenza «facendo leva non solo sulle modalità razionali e cognitive ma innescando una relazione emozionale indispensabile per costruire quel ponte necessario per una comunicazione attiva e partecipativa» (Ippoliti \& Casale, 2018, p. 130);

\footnotetext{
${ }^{25}$ Per la didattica museale, come per la scuola, l'uso delle ICT perde di senso e di efficacia se si limita alla riproposizione di lezioni frontali (Biondi, 2007, p. 61).

${ }^{26}$ Sulle potenzialità delle risorse digitali nell'apprendimento collaborativo Guglielman, 2012 pp. 41-42.
}

- favorire le attività di rielaborazione del patrimonio museale e di condivisione, utilizzando la modalità partecipativa della Rete.

Per realizzare prodotti digitali aderenti alla mission del museo, interattivi e accessibili (Chiuppes, 2016) è naturalmente necessario prevedere una stretta sinergia tra il personale scientifico del museo, il responsabile dei servizi educativi, esperti nella comunicazione digitale e nella realizzazione ed ottimizzazione di risorse digitali, le cui competenze andranno di volta in volta declinate sulla base della complessità dei contenuti da realizzare ${ }^{27}$.

Resta comunque innegabile che, sia pure in modo improvvisato, i musei si sono aperti alla comunicazione multimediale. Alcuni prodotti digitali, nati dalla necessità di trovare soluzioni alternative alle attività in presenza, si sono rivelati utili strumenti, funzionali alla preparazione (pre-fruizione) o approfondimento della visita (post-fruizione culturale). Citiamo ad esempio il ciclo di conferenze "Mezz'ora d'arte" proposto dai Musei Civici Fiorentini e MUS.E con il proposito di offrire esperienze virtuali che «lungi dal replicare una visita fisica [...] prenderanno avvio da ciò che il mondo digitale offre, ovvero osservazioni iper ravvicinate, comparazioni, confronti, connessioni tematiche e stilistiche, ricostruzioni digitali» ${ }^{28}$.

Il critico periodo dell'emergenza sanitaria ha portato alcune istituzioni ad investire nelle piattaforme digitali o sviluppare progetti già in atto di aggiornamento dell'infrastruttura tecnologica del museo, come il progetto "Cosmo Digitale" del Castello di Rivoli ${ }^{29}$ o "è Reale. Il canale dei Musei Reali di Torino"30.

La chiusura forzata, ma anche l'inevitabile cambiamento almeno sul breve e medio periodo di accesso ai luoghi di cultura, di organizzazione degli eventi pubblici, di sostenibilità del turismo hanno ribadito l'urgenza del confronto da parte dei musei con nuove strategie comunicative, la necessità di interpretare il web non solo come spazio virtuale dove inserire informazioni pratiche, ma ampliare le azioni del museo e fornire nuovi percorsi di fruizione, conoscenza e rielaborazione del patrimonio (Di Pietro, 2018). La potenza e la necessità dell'interconnessione durante la pandemia Covid-19 si dovranno riflettere nelle scelte dei musei dal momento che "As an institution continuingly reflecting the society in which is located, the relationship a museum has with

\footnotetext{
${ }^{27}$ Il tema delle diverse figure professionali che operano nelle tecnologie digitali e delle loro competenze è quanto mai attuale e complesso: Mastronardi et al., 2017; Silvaggi et al., 2016; Confetto \& Siano, 2017.

28 https://cultura.comune.fi.it/system/files/2020-05/cs\%20 Mezz\%27ora\%20d\%27arte.\%20Conferenze\%20online\%20su\%20ZOOM. pdf, $29 / 08 / 2020$.

${ }^{29} \mathrm{https} / / /$ www.castellodirivoli.org/mostra/cosmodigitale/, 29/08/2020.

${ }^{30} \mathrm{https} / / /$ www.museireali-ereale.it/?cn-reloaded=1, 29/08/2020.
} 
its community is continually being re-negotiated and reinvented» (Shahani et al., 2008, p. 19).

\section{RIFERIMENTI BIBLIOGRAFICI}

Antinucci, F. (2007). Musei virtuali. Come non fare innovazione tecnologica. Laterza.

Biondi, G. (2007). La scuola dopo le nuove tecnologie. Apogeo.

Bonacasa, N. (2011). Il museo on line. Nuove prospettive per la museologia, (OADI Digitalia), Osservatorio per le Arti Decorative in Italia "Maria Accascina". http:// www1.unipa.it/oadi/digitalia/01_bonacasa.pdf.

Caprara, B., Colombi, A., \& Scala, C . (2018). I musei italiani nel Web: analisi, riflessioni e proposte didattiche. In A. Luigini, \& C. Panciroli (A cura di), Ambienti digitali per l'educazione allarte e al patrimonio (pp. 79-96). Franco Angeli.

Chiuppes, M. (2016). Musei virtuali e inclusione sociale, Rivista Trimestrale di Scienza dell'Amministrazione, 1, 1-15.

Ciocca, A. (A cura di) (2006). Glossario: Didattica museale e nuove tecnologie, Rassegna dell'Istruzione, 34-63.

Confetto, M.G., \& Siano, A. (2017). Museo e tecnologie digitali: profili professionali emergenti / Museums and digital technologies: emerging professional profiles, Il Capitale culturale, 15, 103-135.

Colombo, M.E. (2020). Musei e cultura digitale fra narrativa, pratiche e testimonianze. Editrice bibliografica.

Dipace, A. (2012). La visita al museo come occasione per sviluppare apprendimenti significativi. Riflessione e spunti per la valutazione. In P. Limone (A cura di), Educazione, scuole e musei. Un progetto collaborativo di innovazione didattica, Collana di studi e ricerche sulla cittadinanza digitale 3, (pp. 121-136). Carocci.

Di Pietro, I. (2018). Il sito web museale: quali obiettivi per la comunicazione digitale?. In A. Luigini, \& C. Panciroli (A cura di), Ambienti digitali per l'educazione allarte e al patrimonio (pp. 307-322), Franco Angeli.

Guglielman, E. (2004). Le tecnologie digitali per la didattica tra innovazione e nuove competenze, Life Design - Research and Education, (II) 2, 38-46.

Ippoliti, E., \& Casale, A. (2018). Rappresentare, comunicare, narrare. Spazi e musei virtuali tra riflessioni e ricerche. In A. Luigini, \& C. Panciroli (A cura di), Ambienti digitali per leducazione allarte e al patrimonio (pp. 128-150). Franco Angeli.

ISTAT (2019a). I musei, le aree archeologiche e i monumenti in Italia anno 2017. https://www.journalchc.com/wp-content/uploads/2019/02/reportmusei_2017.pdf.

ISTAT (2019b). L'Italia dei Musei, Statistiche today.
https://www.istat.it/it/files/2019/12/LItalia-deimusei_2018.pdf.

Jalla, D., Lattanzi, V., Maffei, T., Mandosi, M., Maresca Compagna, A., Mascheroni, S., Sodano, C., Visser, A.M., \& Marras, A.M. (A cura di) (2017). Quaderno per la riforma n. 2 del novembre 2017 - Professionalità e funzioni del museo alla luce della riforma dei musei statali, ICOM Italia.

Lin, A.C.H., \& Gregor, S. (2006). Designing Websites for Learning and Enjoyment: A study of museum experiences, International Review of Research in Open and Distance Learning, (7) 3, 1-21.

Mastronardi, G., Santoni, A., Rapari, G., Rigoni, L. (2017). Osservatorio delle competenze digitali 2017. Scenari, gap, nuovi profili professionali e percorsi formativi. Grafiche Ancora. https://www.agid.gov.it/ sites/default/files/repository_files/osservatorio_competenze_digitali_2017.pdf.

Panzeri, M. (2010). Interfacce Internet dei musei d'arte in Italia: presupposti di una prospezione, Digitalia. Rivista del digitale nei beni culturali, (V) 1, 67-94.

Shahani, L., Economou, M., \& Nikonakou, M., (2008). Museums curating online content using Web2.0: making cultural production more democratic?, paper presented in The digital Curation of Cultural Heritage 2008. Annual Conference of CIDOC, Athens, September 12-18 2008. http://Cidoc-mediahost. org/content/archive/cidoc2008/Documents/papers/ drfile.2008-06-38.pdf

Schnapp, J.T. (2012). Musei 2.0. In P. Limone (A cura di), Educazione, scuole e musei. Un progetto collaborativo di innovazione didattica, Collana di studi e ricerche sulla cittadinanza digitale 3, (pp. 16-29). Carocci.

Silvaggi, A., Pesce, F., \& Surace, R. (2016), Musei del futuro. Competenze digitali per il cambiamento $e$ l'innovazione in Italia. Report progetto MuSA-Museum Sector Alliance. http://www.project-musa.eu/ it/wp-content/uploads/sites/5/2018/03/Musei-delfuturo_Mu.SA-it.pdf

Valentino, P.A., \& Delli Quadri, M.R. (A cura di) (2004). Cultura in gioco. Giunti.

\section{SITOGRAFIA}

Castello di Rivoli (n.d.). Cosmo digitale. https://www.castellodirivoli.org/mostra/cosmodigitale/

Comune di Firenze (2020, maggio). Mezzora d'arte Conferenze digitali per esplorare le bellezze dei musei civici fiorentini. https://cultura.comune.fi.it/system/ files/2020-05/cs\%20Mezz\%27ora\%20d\%27arte.\%20 Conferenze\%20online\%20su\%20ZOOM.pdf 
Fondazione Brescia Musei (n.d.). Il Museo in classe. Visite virtuali e approfondimenti sulla storia e l'arte al servizio della scuola. https://didattica.bresciamusei. com/pag.asp? $\mathrm{n}=29 \& \mathrm{t}=$ Spazio + Scuola

Fondazione Ivan Bruschi (2020, 13 marzo). Ogni cosa da noi e' una storia...\#noixvoi. http://www.fondazioneivanbruschi.it/eventi/cosa-storia-noidavoi/

Fondazione Museo Civico di Rovereto (n.d.). Tutti i video per la scuola. http://www.fondazionemcr.it/context. jsp?ID_LINK $=114890 \&$ area $=279$

Galleria degli Uffizi (2020, 10 marzo). Uffizi Decameron in rete! https://www.uffizi.it/magazine/uffizi-facebook- 2020

ICOM (2020, 30 giugno). Comunicazione digitale dei musei ai tempi del covid-19. http://www.icom-italia. org/comunicazione-digitale-dei-musei-ai-tempi-delcovid-19/

Musei Civici di Monza (n.d.). Gioca Museo...da casa! [Video] http://www.museicivicimonza.it/2020/04/27/ iorestoacasagiocamuseo-da-casa

Musei Reali di Torino (n.d.). È reale: il canale dei Musei Reali. https://www.museireali-ereale.it/?cn-reloaded $=1$

MUS.E Firenze (n.d.). Museo chiama casa - speciale bambini. http://musefirenze.it/museo-chiama-casa-speciale-bambini/

MUSE Museo delle Scienze. (2020, 17 marzo). Introduzione a "Quattro passi nella Preistoria. [Video] YouTube. https://www.youtube.com/watch?v=ICZu6MFqIoA\&li st=PLS5GAt5gHhU_JXYcGMBQrFkADR3wPJKFp

Museo Archeologico Nazionale di Aquileia (n.d.). \#il museo a casa. https://museoarcheologicoaquileia. beniculturali.it/ilmuseoacasa/\#page-content

Museo Egizio Torino (n.d.). Il Museo Egizio a casa tua. https://museoegizio.it/esplora/notizie/il-museoegizio-a-casa-tua/

Museo Galileo Istituto e Museo di Storia della Scienza (n.d.). Impara: Online. https://www.museogalileo.it/it/ museo/impara/online.html

Museo Nazionale delle Arti del XXI secolo (n.d.). \#iorestoacasa con il MAXXI. https://www.maxxi.art/ iorestoacasa/

Museo Nazionale della Scienza e della Tecnologia Leonardo da Vinci (2020, 25 febbraio). Storie a porte chiuse. https://www.museoscienza.org/it/storieaportechiuse

Museo Paleontologico di Montevarchi (2020, 19 marzo). Visita il Museo...da casa! http://www.museopaleontologicomontevarchi.it/2020/03/19/visita-il-museo-dacasa/

Museo Poldi Pezzoli (2020,16 marzo). \#PoldiPezzoliStories. https://museopoldipezzoli.it/tutti-gli-eventi/poldipezzolistories/
Pinacoteca di Brera (n.d.). Brera Media. https://pinacotecabrera.org/brera-media/

Sa Rundine, Museo Archeologico Paleobotanico di Perfugas (n.d.). MAP: visita il MAP da casa. https://www. sarundine.com/approfondimenti-video/

Senza Titolo (n.d.). ST senza titolo: progetti aperti alla cultura. https://www.senzatitolo.net/in-corso/316/ didattica_a_distanza_tra_scuola_e_museo:_proposte_ educative_in_digitale_per_larte_contemporanea_/

Università degli Studi di Firenze, Sistema Museale di Ateneo (n.d.). Tempo di Giochi. https://www.sma.unifi.it/ p609.html 